EPJ direct (2018), to be processed by the editorial

EPJ direct

electronic only

(C) Springer-Verlag 2018

\title{
Pictures of quantum nuclear rotation beyond the correspondence principle
}

\author{
R. Arvieu ${ }^{1}$, P. Rozmej ${ }^{2,3}$ \\ 1 Institut des Sciences Nucléaires, 53 av. des Martyrs, F-38026 Grenoble-Cedex, France \\ e-mail: arvieu@in2p3.fr \\ 2 Theoretical Physics Department, University Maria Curie-Skłodowska, PL-20031 Lub- \\ lin, Poland \\ e-mail: rozmej@tytan.umcs.lublin.pl \\ 3 Gesellschaft für Schwerionenforschung mbH, Planckstr. 1, D-64291 Darmstadt, Ger- \\ many \\ e-mail: p.rozmej@gsi.de
}

\begin{abstract}
We analyze the time evolution of simple nuclear rotational wave packets (WP) called circular, linear or elliptic, depending on squeezing parameter $\eta$, assuming that $E=\hbar \omega_{0} I(I+1)$. The scenario of fractional revivals found by Averbukh and Perelman is adapted to symmetric WP and compared to that which holds for asymmetric WP. In both cases various shapes are identified under these lines in particular many cases of cloning. 'Mutants' WP are found most often. Finally the time evolution of a WP formed by Coulomb excitation on ${ }^{238} \mathrm{U}$ and calculated by semiclassical theory is also presented.
\end{abstract}

PACS: 03.65.Sq, 03.65.Ge, 21.10Re, 21.10Ev

\section{Introduction}

It is somewhat paradoxical after many years of confirmation of quantum mechanics that new features can be learned on two of the simplest systems that are taught in the elementary courses: the hydrogen atom and the rigid rotor. It was indeed realized only in the early 90's [1, 2] that a wave packet in the hydrogen atom evolves in time according to a universal scenario discovered theoretically by Averbukh and Perelman [3]. For short times a well built wave packet moves around a Kepler ellipsis, and then it spreads along the ellipsis. The surprise was that the wave packet is seen at specific times as a superposition of fractional wave packets concentrated around specific points on this trajectory. This behaviour results from the unitary quantum evolution and from self interferences. By changing the initial wave packet one can also obtain a radial wave packet without an analogue classical trajectory which will also experience the scenario of fractional revivals 细. An extension of these results has later been been made by Bluhm and Kostelecky [5] who showed the existence of superrevivals for longer times.

The aim of this article is to describe the similar steps for the rotation of symmetric rigid rotors with possible application to nuclei and to molecules. The 
theory of ref. [3] is exact for all times if the energy spectrum is quadratic in one quantum like the infinite square well [6] or in two quantum numbers like [7] or the rigid top with axial symmetry. In ref. [8] we studied various sets of angular momentum coherent states which represent an heteronuclear diatomic molecule or a rigid body with axial symmetry. Our purpose is to show the rotation of a symmetric object described by a quantum pure state in the most obvious scheme (i.e. if the law $E=\left(\hbar^{2} / 2 J\right)(I(I+1))$ holds exactly) for a wave packet specifically designed to rotate and what the motion is, if the initial preparation of the wave packet is different. Because of the quantum spreading a quantum WP does not rotate at all like a classical rigid body, its spreading differs according to the initial conditions of its preparation. The introduction of this spreading is necessary if one introduces the concept of a WP which is not a common practice in the field of rotational nuclei or molecules. We must however acknowledge that this concept was first introduced in the past in the reference book by Broglia and Winther [9] on heavy ions collisions, then in a more extensive work by Fonda et. al. [10] who have discussed the mechanism of generation of several sets of rotational coherent WP. Our work completes these two works in the sense that we give a full study of the time evolution of the WP which is now possible after the work of [3]. Our WP depend also on a new squeezing parameter $\eta$ introduced in [8] which enables to change the geometry of the WP in a most significant manner.

Before proceding to our work we must also quote most elegant papers by Berry and Golberg [11] and Berry [12] who have studied the full time evolution of the propagator of a nuclear spin with a hamiltonian of the form $L^{2} /(2 J)$. More recently various Talbot effects were also discovered with the help of renormalization techniques 13]. The occurrence of fractal dimensions both in space and time during the evolution of uniform WP in boxes of arbitrary dimensions has been also discovered 14. These last results show that the spreading of WP in a bound system is extremely rich. Our purpose is to emphasize this richness for two dimensional WP on the sphere.

\section{Asymmetric and symmetric squeezed WP}

\subsection{Asymmetric WP}

In ref. [8] we have studied the properties and the time evolution of the following WP called the exponential coherent WP which depends on two parameters $N$ and $\eta$. This normalized state is defined as

$$
\Psi_{\eta}(\theta, \phi)=\sqrt{\frac{2 N}{4 \pi \sinh (2 N)}} \mathrm{e}^{N \sin \theta(\cos \phi+\mathrm{i} \eta \sin \phi)} .
$$

For large enough values of $N$ the probability density associated to it is symmetric around $O x$ and is peaked within a solid angle $\Omega=4 \pi /(4 N+1)$. Thus, large values of $N$ correspond to defining the orientation of the axis of symmetry of the system in a very sharp way, quite independently on the value of the average angular momentum $\mathbf{L}$. For the components of $\mathrm{L}$ one can see that

$$
\left\langle L_{x}\right\rangle=0 \quad\left\langle L_{y}\right\rangle=0,
$$




$$
\left\langle L_{z}\right\rangle=\eta\left(N-\frac{1}{2}\right)
$$

Equation (3) is correct only for large $N$, for small $N$ there is a small correction term not important for our discussion. Therefore, the average of $\mathbf{L}$ is fixed by the product $\eta N$. Finally we have proved [8] that the state (11) is made of a special class of angular momentum states called in the literature by the name of intelligent spin states (for real values of $\eta$ ). They obey the equation

$$
\left(L_{x}+\mathrm{i} \eta L_{y}\right) \Psi_{\eta+}=0 .
$$

From this equation one can derive that the parameter $\eta$ has the meaning of a squeezing parameter since

$$
|\eta|^{2}=\frac{\Delta L_{x}^{2}}{\Delta L_{y}^{2}}
$$

For all real values of $\eta$ the minimum uncertainty condition is satisfied

$$
\Delta L_{x}^{2} \Delta L_{y}^{2}=\frac{1}{4}\left\langle L_{z}\right\rangle^{2} .
$$

The states (11) are such the analogous of the squeezed states of the electromagnetic field. In this article we will distinguish two limiting values of $\eta$ : the case $\eta=0$ corresponds to a purely real WP initially without momentum which we call the linear WP, the case $\eta=1$, called a circular WP, is an eigenstate of $L_{+}$. The other values correspond in the same terminology [8] to elliptic WP. Indeed these WP can be derived from Gaussian WP which are respectively associated to linear, circular and elliptic trajectories if they evolve in 3D harmonic oscillator.

The case of complex values of $\eta$ does not bring any interesting freedom [17], the main difference lying in the fact that the equality (6) is violated. Therefore $\eta$ will take only real values in the following.

The spherical harmonic expansion of the state (1) is written in terms of coefficients $b_{I M}$ as

$$
\Psi_{\eta}=\sum_{I M} b_{I M} Y_{M}^{I}(\theta, \phi)
$$

$$
\begin{aligned}
b_{I M}= & \sqrt{\frac{2 N}{\sinh (2 N)}} \\
& \times \sum_{l l^{\prime}}(-1)^{l^{\prime}} N^{l+l^{\prime}} \frac{(1+\eta)^{l}(1-\eta)^{l^{\prime}}}{\sqrt{(2 l) !\left(2 l^{\prime}\right) !}} \frac{\left\langle l l^{\prime} 00 \mid I 0\right\rangle\left\langle l l^{\prime} l-l^{\prime} \mid I M\right\rangle}{\sqrt{(2 I+1)}} .
\end{aligned}
$$

For $\eta=1$ only the $b$ 's with $M=I$ are non-zero. This is why these wave packets are called circular. In the case $\eta=0$ it is simpler to make a rotation of the coordinate axis to get an eigenstate of $L_{z}$ with eigenvalue 0 , the expansion of $\Psi_{0}$ takes the form

$$
\Psi_{0}=\sum_{I} b_{I 0} Y_{0}^{I}(\theta, \phi) .
$$

with $b_{I 0}$ given in terms of spherical Bessel function of the first kind by

$$
b_{I 0}=\sqrt{\frac{2 N}{\sinh (2 N)}} \sqrt{(2 I+1)} \sqrt{\frac{\pi}{2 N}} I_{I+1 / 2}(N) .
$$




\subsection{Symmetric WP}

The situation we want to describe is that of an even, deformed nucleus with an axis of symmetry $O_{z}$ such that $\left\langle L_{Z}\right\rangle=K=0$ and with a plane of symmetry perpendicular to this axis. No other degree of freedom will be included. We therefore consider instead of (11) the following symmetrical combination

$$
\Psi_{\eta+}(\theta, \phi)=\frac{C_{+}}{2}\left[\mathrm{e}^{N \sin \theta(\cos \phi+\mathrm{i} \eta \sin \phi)}+\mathrm{e}^{-N \sin \theta(\cos \phi+\mathrm{i} \eta \sin \phi)}\right] .
$$

Clearly for large $N$ it represents two antipodal waves, within the same solid angle as above and with the same symmetry around $O x$. The normalization constant $C_{+}$depends now both on $N$ and $\eta N$ because of an interference term between the two waves. It is given by

$$
C_{+}=\left\{2 \pi\left[\frac{\sinh 2 N}{2 N}+\frac{\sin 2 \eta N}{2 \eta N}\right]\right\}^{(-1 / 2)}
$$

It should be stressed that the main difference in the time evolution of (11) and (11) comes from the interference between these two parts. As we will see it will become much different from a normalization factor.

It is straightforward to verify that eqs. (2) to (6) are still valid. We now express the wave packet $\Psi_{\eta+}$ in terms of new coefficients $b_{I M+}$ as

$$
\Psi_{\eta+}=\sum_{I M} b_{I M+} Y_{M}^{I}(\theta, \phi)
$$

and these coefficients are related to the $b_{I M}$ by

$$
b_{I M+}= \begin{cases}0 & \text { for } I \text { odd } \\ \sqrt{2}\left(1+\frac{\sin 2 \eta N}{\eta \sinh 2 N}\right)^{-1} b_{I M} \simeq \sqrt{2} b_{I M} & \text { for } I \text { even. }\end{cases}
$$

\section{Time evolution}

\subsection{The rigid rotor assumption}

Let us study the evolution of $\Psi_{\eta+}$ for $\eta=0$ and $\eta=1$ assuming that the energy levels obey the rule

$$
E_{I}=\frac{\hbar^{2}}{2 J} I(I+1)
$$

In the following we will use $\omega_{0}=\hbar /(2 J)$ and we define an average angular momentum by

$$
\bar{I}(\bar{I}+1)=\left\langle\Psi_{\eta+}\left|L^{2}\right| \Psi_{\eta+}\right\rangle .
$$

We can now use the work of [3] to analyze the structure of the wave packets at time $t$ knowing simply that the wave packets are given by

$$
\Psi_{\eta+}(\theta, \phi, t)=\sum_{I M} b_{I M+} Y_{M}^{I}(\theta, \phi) \mathrm{e}^{-\mathrm{i} \omega_{0} I(I+1) t}
$$


It is necessary to introduce two characteristic times as in [3]

$$
T_{\mathrm{cl}}=\frac{2 \pi}{\omega_{0}} \frac{1}{2 \bar{I}+1}, \quad \quad T_{\mathrm{rev}}=\frac{2 \pi}{\omega_{0}} .
$$

Note that $T_{\text {rev }}>>T_{\mathrm{cl}}$ if $\bar{I}$ is large enough. We have with (17) and (18)

$$
\Psi_{\eta+}\left(\theta, \phi ; T_{\mathrm{rev}}\right)=\Psi_{\eta+}(\theta, \phi ; 0) .
$$

\subsection{The outline of the theory of fractional revivals}

Let us sketch the general theory of [3] which allows to find a universal scenario of fractional revivals. Near $t=(m / n) T_{\text {rev }}$, where $m / n$ is an irreducible ratio of two integers, it has been proved in [3] that

$$
\mathrm{e}^{-2 \mathrm{i} \pi I^{2} \frac{m}{n}}=\sum_{s=0}^{l-1} a_{s} \mathrm{e}^{-2 \mathrm{i} \pi I \frac{s}{l}}
$$

This sum contains $q=\frac{n}{2}$ non-zero terms if $n$ is even and $q=n$ terms if $n$ is odd. The integer $l=\frac{n}{2}$ if $n$ is a multiple of $4, l=n$ in the other cases (therefore the difference between $l$ and $q$ is necessary to take into account that the $a_{s}$ with even $s$ are zero if $n$ is even and not multiple of 4). The modulus of $a_{s}$ is simply for all the cases where it is non-zero equal to:

$$
\left|a_{s}\right|=\frac{1}{\sqrt{q}}
$$

The phase of $a_{s}$ as a function of $m, n$ and $s$ can be found in an older paper written in a quite different context 15]. Therefore one writes after the insertion of (20) into (7) taken at time $t=\frac{m}{n} T_{\text {rev }}$

$$
\begin{aligned}
\Psi_{\eta}\left(\theta, \phi ; \frac{m}{n} T_{\mathrm{rev}}\right) & =\sum_{s=0}^{l-1} a_{s}\left[\sum_{I M} b_{I M} Y_{M}^{I} \mathrm{e}^{-2 \mathrm{i} \pi I\left(\frac{m}{n}+\frac{s}{l}\right)}\right] \\
& =\sum_{s=0}^{l-1} a_{s} \Psi_{\mathrm{cl}}^{(s)}\left(\theta, \phi ; t_{s}\right) .
\end{aligned}
$$

Any wave packet is a sum of $q$ fractional wave packets $\Psi_{\mathrm{cl}}^{(s)}$, each with a different effective time $t_{s}$

$$
t_{s}=\left(\frac{m}{n}+\frac{s}{l}\right) T_{\mathrm{rev}} .
$$

The fractional wave packet is defined as

$$
\Psi_{\mathrm{cl}}^{(s)}\left(\theta, \phi ; t_{s}\right)=\sum_{I M} b_{I M} Y_{M}^{I} \mathrm{e}^{-\mathrm{i} \omega_{0} I t_{s}} .
$$




\subsection{Clones and mutants}

There are several cases for which $\Psi_{\mathrm{cl}}$ is a clone of the initial $\Psi$ defined by (1) or (7):

i) if the expansion (7) contains only $b_{I M}$ with the single values $M=I$, this is the case for the wave packet (11) for $\eta=1$. One obtains for each $t_{s}$

$$
\Psi_{\mathrm{cl}}\left(\theta, \phi ; t_{s}\right)=\Psi_{\eta}\left(\theta, \phi-\omega_{0} t_{s}\right) .
$$

This result is independent of the distribution of the $b_{I I}$ 's, it applies to all kinds of circular wave packets. The sum (23) is a superposition of $q$ clones of the initial wave packet. In 6 this conclusion was derived for an infinite one-dimensional well. In our case with $\eta=1$ the $q$ clones are localised around $q$ axis forming a symmetric fan in the $O x y$ plane.

ii) if $\omega_{0} t_{s}$ is a multiple of $2 \pi$, i.e. if $a_{s_{0}}$ is non-zero for the value

$$
s_{0}=n-m,
$$

then

$$
\Psi_{\mathrm{cl}}\left(\theta, \phi ; t_{s_{0}}\right)=\Psi_{\eta}(\theta, \phi, 0) .
$$

This occurs when $n$ is odd or even and not multiple of 4 . For these times there is a clone at the place of the initial wave packet with the amplitude $a_{s_{0}}$. The existence of this clone is independent of the $b_{I M}$ and the parameter $\eta$.

In [8] we have proposed to call mutants the fractional wave packets $\Psi_{\mathrm{cl}}$ which are not clones and which have a different geometry due to the phase $\mathrm{e}^{-\mathrm{i} \omega_{0} I t_{s}}$ in (25). We have also described the change of the shape of mutants as a function of $\eta$ for fixed $t_{s}$. This is made by considering two extreme cases where the shape of $\Psi_{\mathrm{cl}}$ is simply understood. For $\eta=1 \Psi_{\mathrm{cl}}$ is a clone of the initial wave packet located around a given direction in the $O x y$ plane. For $\eta=0$ the expansion (9) shows that wave packet $\Psi_{0}$ at $t=0$ as well as for all $t$ and also each $\Psi_{\mathrm{cl}}$ have cylindrical symmetry around $O x . \Psi_{\mathrm{cl}}$ is therefore peaked around one or two rings around $O x$. For intermediate values of $\eta$ the mutants have shapes making a smooth transition between the two extreme cases.

This theory fits perfectly to the case of the asymmetric WP which contains terms with even and odd values of $I$ in equations (7), (20) and (22). In the case $\frac{m}{n}=\frac{1}{2}$ eq. (24) gives $t_{s}=T_{\text {rev }}, a_{s}=\delta_{s 1}$ and the WP is periodic with period $t_{s}=T_{\text {rev }} / 2$. This reduced periodicity can be seen immediately from the fact that $I(I+1)$ is always even. It is therefore enough to consider times such that $\frac{m}{n} \leq \frac{1}{2}$.

\subsection{Additional rules for symmetric WP}

In the symmetric case corresponding to 111$)$ and (13) with (14), an additional symmetry arises from the fact that eq. (20) is now used for even values of $I$. If we put $n=4 n^{\prime}$ in (20) we obtain $q=l=2 n^{\prime}$ values of $s$ in the general case. 
However if $I$ is always even $l$ takes the value of $n^{\prime} / 2$ if $n^{\prime}$ is a multiple of 4 or $n^{\prime}$ is odd or even and not multiple of 4 . For $n^{\prime}$ even and not multiple of 4 the $a_{s}$ with even $s$ are zero. Therefore the number $q$ of fractional waves is largely reduced and becomes

$$
q= \begin{cases}n^{\prime} & \text { for odd } n^{\prime} \\ n^{\prime} / 2 & \text { for even } n^{\prime}\end{cases}
$$

For $n^{\prime}=1$ one can write

$$
\mathrm{e}^{-2 \mathrm{i} \pi I(I+1) / 4}=\mathrm{e}^{\mathrm{i} \pi I / 2},
$$

for $n^{\prime}=2$

$$
\mathrm{e}^{-2 \mathrm{i} \pi I(I+1) / 8}=\mathrm{e}^{-\mathrm{i} 3 \pi I / 4} .
$$

Inserting these values in (17) for the particular case of circular WP $(\eta=1)$ one obtains the following periodic behaviour

$$
\begin{gathered}
\Psi_{1+}\left(\theta, \phi, T_{\text {rev }} / 4\right)=\Psi_{1+}(\theta, \phi+\pi / 2,0), \\
\Psi_{1+}\left(\theta, \phi, T_{\text {rev }} / 8\right)=\Psi_{1+}(\theta, \phi-3 \pi / 4,0) .
\end{gathered}
$$

For $\eta \neq 1$ the insertion of the phases (29) into (17) produces a single WP different from the initial one, i.e. a single mutant. For $\eta=1$ the time interval necessary to study the evolution is $t=T_{\text {rev }} / 8$, but for $\eta \neq 1$ it is still $t=T_{\text {rev }} / 2$.

At fractional times of $T_{\text {rev }} / 4$, like $t=T_{\text {rev }} \frac{m}{4 n^{\prime}}$ the following formula holds for $\eta=1$ and describes fully the cloning of these species of symmetric WP

$$
\Psi_{1+}\left(\theta, \phi, T_{\mathrm{rev}} \frac{m}{4 n^{\prime}}\right)=\sum_{s=0}^{l} a_{s} \Psi_{1+}\left(\theta, \phi-\pi\left(\frac{s}{l}+\frac{m}{2 n^{\prime}}\right), 0\right),
$$

with the general result that the sum contains $q=n^{\prime} / 2$ non-zero terms for even $n^{\prime}$ (with $l=n^{\prime} / 2$ if $n^{\prime}$ is a multiple of $4, l=n^{\prime}$ in other cases and $a_{s}$ is zero if $n^{\prime}$ is even and not multiple of 4 and $s$ is even).

For example the application of this formula for $t=T_{\text {rev }} / 16$ gives two clones oriented along perpendicular directions in the $O x y$ plane since

$$
\Psi_{1+}\left(\theta, \phi, T_{\mathrm{rev}} / 16\right)=a_{0} \Psi_{1}(\theta, \phi-\pi / 8,0)+a_{1} \Psi_{1}(\theta, \phi-5 \pi / 8,0) .
$$

Similarly, for $t=T_{\text {rev }} / 24$ one obtains 3 clones etc... For $\eta \neq 1$ the clones should be replaced by a corresponding number of mutants.

If on the other hand $n$ is even and not multiple of 4, i.e. if $n=2 n^{\prime}$ with $n^{\prime}$ odd, the limitation of even $I$ in eq. 20) leads to $l=n^{\prime}$ therefore all the $a_{s}$ are non-zero but should be calculated with an effective ratio $2 m / n^{\prime}$ instead of $m / n$. The number of fractional WP is then the same as for the asymmetric case. Similarly, if $n$ is odd there are $q=n$ fractional WP. Note that for odd $n$ and even $n$ not multiple of 4 eq. (27) and (28) also hold: one of the fractional WP is a clone at the place of the initial WP. 


\section{Numerical calculations for the exponential coherent WP.}

\subsection{Comparisons between the symmetric and asymmetric WP}

As already stated above, the WP (11) differs from (11) by the interference terms which are almost negligible if one calculates the probability density at $\mathrm{t}=0$. However the previous discussion on the time evolution shows a large number of differences. Therefore a comparison between asymmetric and symmetric WP is presented in the figures of this letter. With high enough $N$ one obtains a generic behaviour. For $\eta=1$ we have chosen $N=14$ i.e. $\bar{I}=13.5$. This value of $\bar{I}$ corresponds to the average excitation energy of the $K=0$ rotational band of ${ }^{238} \mathrm{U}$ that is obtained from Coulomb excitation by $200 \mathrm{MeV}{ }^{40} \mathrm{Ar}$ projectiles. Coulomb excitation does not produce weights $b_{I M}$ so simply distributed to give a WP like (11).

Fig. 11 shows 'carpets' for the two cases with $N=14$ and $\eta=1$. The carpet is the intersection of the plane $O x y$ with the probability density as a function of time for $0<t<T_{\text {rev }} / 2$. For $t<T_{\text {rev }} / 25=T_{\mathrm{cl}}$ the WP spread and occupy all values of phi. For $t>T_{\mathrm{cl}}$ there are clear windows near values $(m / n) T_{\text {rev }}$ for which the WP is formed of $q$ clones, with $q$ given as discussed in the text for asymmetric and symmetric case respectively. In the former case one has large windows for $m / n=1 / 4(q=2), m / n=1 / 6$ and $1 / 3(q=3), m / n=1 / 8$ and $3 / 8(q=4)$ and it is possible to see as much as $q=8$ clones without much interference between them (see Fig. 2). In the latter case we obtain instead due to eqs. (31) and (33) a slide angle of $-3 \pi / 4$ since the following equation takes place

$$
\Psi_{1+}\left(\theta, \phi, t+T_{\text {rev }} / 8\right)=\Psi_{1+}(\theta, \phi-3 \pi / 4, t) .
$$

Therefore the interval $T_{\text {rev }} / 2$ is separated into four stripes into which essentially only a single large time window is seen for $m / n=1 / 16$ with $q=n^{\prime} / 2=2$ clones. By zooming within $T_{\text {rev }} / 8$ one can see other windows as well as we will see in Fig. 3.

Fig. 2 and 3 show the probability density as a function of $\theta$ and $\phi$ for a selection of times where one can underline similarities or differences between the two cases. For $0<t<T_{\mathrm{cl}}$ the WP essentially spreads in $\phi$ because the corresponding values of $n$ produce too many clones with big interferences [6]. For $t=T_{\text {rev }} / 50$ the two peaks forming the symmetric WP interfere strongly and the WP occupies already all the values of $\phi$. In the next sequences of times one identifies $\left(q, q^{\prime}\right)$ clones respectively in the asymmetric and symmetric cases: $m / n=1 / 16(8,2), m / n=1 / 3(3,3), m / n=1 / 24(12,3)$ : there the 12 clones are strongly interfering, $m / n=1 / 5(5,5), m / n=1 / 6(3,3)$.

Fig. A shows the carpets corresponding to $\eta=0$ with $N=110$ and not $N=14$. The distribution of the $b_{I M}$ with $I$ depends indeed very much on the value of $\eta$. Using $N=14$ with $\eta=0$ produces too low value of $\bar{I}$ and a rather poor carpet. The value $N=110$ corresponds to $\bar{I}=10$ and leads to richer figures. For this value of $\eta$ the WP is axially symmetric for all times, the carpet is therefore defined as the quantity $2 \pi \sin \theta\left|\psi_{0}(\theta, t)\right|^{2}$ as a function of $\theta$ and $t$. Both carpets are also symmetric around $t=T_{\text {rev }} / 4$. However only the carpet 
showing the symmetric case has an additional symmetry around $\theta=\pi / 2$. Indeed a time $\tau$ which fulfills for all I the equation

$$
\exp \left[-\mathrm{i} \omega_{0} \tau I(I+1)\right]=(-1)^{I}
$$

does not exist. The maxima of the $\Psi_{\mathrm{cl}}^{(s)}\left(\theta, t_{s}\right)$ occur for definite values of $\theta$ and are not always distributed symmetrically around $\pi / 2$. These fractional waves can be understood as waves on a sphere with maximum along a ring [8]. For $t=T_{\text {rev }} / 4$ there is a unique large ring at $\theta=\pi / 2$. Moreover, since $b_{I 0}$ and $Y_{I 0}$ are both real, values $s$ and $s^{\prime}$ may exist such that

$$
\Psi_{\mathrm{cl}}^{\left(s^{\prime}\right)}=\Psi_{\mathrm{cl}}^{(s)^{*}},
$$

for example for $t=1 / 10 T_{\text {rev }}$ there are 5 fractional WP with $0<s<4$. For $s_{0}=n-m=4$ there is a clone such that $\Psi_{\mathrm{cl}}^{(4)}$ is equal to the initial $\Psi$. This WP is represented as a dark dot at $\theta=0$ in the left part of Fig. 1t and there are two pairs of fractional WP, one for $s=0$ and $s^{\prime}=4$, the other for $s=2$ and $s^{\prime}=3$. By addition of these $4 \mathrm{WP}$ one obtains only two rings represented as two other large dots for different $\theta$. In the symmetric case this number is doubled for $T_{\text {rev }} / 10$. As discussed above the existence of the clone relies upon valus of $m / n$.

In the right part of Fig. 1 tone can most often detect a ring at $\theta=\pi / 2$. Similar carpets have been discussed in [16] for the infinite well in one dimension.

\subsection{Geometrical shapes of clones and mutants}

We present in Fig. 目 a short selection of shapes of WP for 4 different values of $\eta$ and the same time $t=T_{\text {rev }} / 8$. Only the part of the $|\Psi|^{2}$ with $z>0$ is shown, note that $\phi$ is defined as the angle with the $O y$ axis. In the asymmetric case one obtains 4 clones for $\eta=1$. When $\eta$ is decreased these clones become 'mutants' which develop a larger spread in the angle $\theta$. For $\eta=0$, as well as for $\eta=1 / 25$, one obtains two rings around the symmetry axis $O y$ as well as a maximum along this axis. In the symmetric case we illustrate for the circular WP the law given by eqs. (33.36): there is a periodicity with a slide angle $-3 \pi / 4$. For different $\eta$ the clone is transformed into a single mutant with a larger spread in $\theta$. For the case of the linear WP this mutant is cylindrically symmetric around $O y$. Note that the mutant has, for $\eta=1 / 25$, an interesting dissymmetrical shape which is necessary in order to make the transition between the clone for $\eta=1$ and the two rings for $\eta=0$.

The WP studied in this letter exibits well defined shapes and a well understood evolution. This is due to the assumptions that the law (15) holds exactly and that the $b_{I M}$ are simply distributed according to (8) in order to correspond to the simple exponentials (11) or (11).

\section{Calculations with realistic WP}

It is quite possible to improve our study in order to incorporate ingredients which make it more realistic. It is well known 9, 10] that during Coulomb excitation 
$(\mathrm{CE})$ a deformed nucleus is excited to a coherent mixture of rotational states. This superposition is also peaked around a mean value of angular momentum, so one can expect similar features as predicted by scenario of Averbukh and Perelman. The most clear case is CE with backscattering as in this case excited WP has cylindrical symmetry (only $Y_{0}^{I}$ components, i.e. $\eta=0$ or linear WP). The partial waves and fractional revivals have then topology of rings on a sphere. Therefore for presentation of shapes only one angular variable $(\theta)$ is sufficient. In Fig. 6 we present the time evolution of WP obtained by $\mathrm{CE}$ of ${ }^{238} \mathrm{U}$ bombarded by ${ }^{40} \mathrm{Ar}$ at $\mathrm{E}=170 \mathrm{MeV}$. The amplitudes of excitation of given $I$ angular momentum eigenstates have been calculated within semiclassical theory of CE [9]. The left part shows the 'ideal case', i.e. when $E_{I}$ 's follow perfect rotor dependence $I(I+1)$, the right corresponds to time evolution with energies taken from experiment. In the latter case it is necessary to define time scales of the evolution. In general [3]

$$
T_{\mathrm{rev}}=\frac{2 \pi}{\frac{1}{2}\left|E_{I}^{\prime \prime}\right|_{I=\bar{I}}}, \quad T_{\mathrm{cl}}=\frac{2 \pi}{\left|E_{i}^{\prime}\right|_{I=\bar{I}}} .
$$

For perfect rotor case these times are determined unambigously: $T_{\mathrm{rev}}=\left(2 \pi / \hbar^{2}\right) J$, $T_{\mathrm{cl}}=T_{\mathrm{rev}} /(2 \bar{I}+1)$. For experimental energies we adopt the following estima-

tion for $T_{\text {rev }}$. The energies of the ground state rotational band for ${ }^{238} \mathrm{U}$ are well approximated by the two parameter formula

$$
E_{I} \simeq a I(I+1)+b[I(I+1)]^{2}
$$

with $a=7.5 \mathrm{MeV}, b=-0.004 \mathrm{MeV}$ [18]. Our 'estimated' $T_{\mathrm{rev}}$, used for the figures (there is no exact revival at all), is then defined by (39) and (40) taken for $\bar{I}$ determined from the equation

$$
\bar{I}(\bar{I}+1)=\left\langle\mathbf{L}^{2}\right\rangle
$$

i.e determined by coefficients of CE rotational WP (see Fig. 8, p. 82 of ref. [9]). In Fig. 7 we display shapes of WP (precisely the probability density as a function of $\theta$ angle) for short times, showing spreading of the WP, initially localized at poles, and clear few rings structure of WP at particular times $\left(3 / 400\right.$ and $\left.4 / 400^{*} T_{\text {rev }}\right)$. In Fig. 8 we show the same WP after much longer evolution, exhibiting structures very similar to those observed for very short times.

Although the 'carpet' for experimental energies isn't as regular as that for 'ideal' ones, still strong revivals of WP occur. The absolute time scales for nuclear rotation are very short $\left(T_{\text {rev }} \sim 10^{-19} \mathrm{~s}, T_{\mathrm{cl}} \sim 10^{-20} \mathrm{~s}\right)$ and are still beyond time resolution of present experimental techniques. It is clear that the picture which emerges from this study underlines that the time evolution of nuclear WP depends strongly on their excitation mechanism as well as from the assumption of a rigid body value for the moment of inertia. The inclusion of more realistic ingredients introduces less symmetry and less regularity in the time evolution.

\section{Conclusions}

There is no doubt that the time evolutions described in our article have been calculated by observing strictly the rules of quantum mechanics for a pure state. 
It is reasonable to believe from the works of $[9]$ and $[10]$ that a WP formed by Coulomb excitation should move as described in section 5 . However there is not much hope that the tools of experimental nuclear physics can evolve in such a way that this time evolution is observable since one needs to probe the WP after its formation with the help of a second projectile. In future the creation of nuclear rotational wave packets and observation of their evolution should be in principle possible with gamma lasers. Nevertheless the picture of the rotation one should have in mind must be that given above, i.e. a picture where the interference effects play a dominant role and differ according to the symmetry of the rotor and its initial preparation.

The field of molecular physics looks more appropriate for observation and many theoretical calculations as well as several experiments have already been realized. Rotational coherence effects of large molecules have been studied theoretically and observed for about ten years [19-21] but are generally understood as thermal average of rotational quantum beats. The rotation of a single molecule of $\mathrm{O}_{2}$ 22 or HB-DC 23 within a supra molecular bearing has been recently observed. The rotation is induced thermally and there is no reason to believe that the system is in a pure state. In the frame of optimal control theory [24. 25], one has also shown recently how to create rotational coherent WP of asymmetric-top molecules by designing tailored microwave pulses 26, 27. The order of magnitude for the time scales of molecular rotations varies from $10^{-12} \mathrm{~s}$ [27 to $10^{-14} s$ [26] and is available for experiment. However, the known excitation mechanisms lead mainly to rovibrational states and an excited superposition is usually composed of smaller number of components than rotational coherent states discussed in this paper. A most important challenge for our purpose would be to find the excitation mechanism that enables the generation of our squeezed WP. Excitation mechanism of a rigid rotor with intense laser field is still under study but like Coulomb excitation of nuclei, this mechanism leads only to linear waves (with $\eta=0$ ).

The study of the time evolution of WP in simple sytems produces behaviour which is full of surprises. A recent work on WP transmitted through a potential barrier has detected the presence of high momentum components which are present during a short time [28] an event which is also a challenge for detection.

Acknowledgements One of us (P. R.) acknowledges financial support from Centre National de la Recherche Scientifique during his stay at ISN.

This work has been supported by KBN Grant No. 2P03B14314.

\section{References}

1. Z. Dačic-Gaeta and C.R. Stroud, Jr., Phys. Rev. A 426308 (1990)

2. S.D. Boris et.al., Phys. Rev. A 482574 (1993)

3. I.S. Averbukh and N.F. Perelman, Phys. Lett. A 139449 (1989)

4. J.H. Yeazell and C.R. Stroud, Jr., Phys. Rev. A 435153 (1991)

5. R. Bluhm and V.A. Kostelecky, Phys. Lett. A 200308 (1995)

6. D.L. Aronstein and C.R. Stroud, Jr., Phys. Rev. A 554526 (1997)

7. G.S. Agarwal and J. Banerji, Phys. Rev. A 573880 (1998)

8. P. Rozmej and R. Arvieu, Phys. Rev. A 584314 (1998) 
9. R. Broglia and A. Winther, Heavy Ion Reactions, (Benjamin, New York, 1981)

10. L. Fonda, N. Mankoč-Borstnik and M. Rosina, Phys. Rep. 158159 (1988)

11. M.V. Berry and J. Goldberg, Nonlinearity 11 (1988)

12. M.V. Berry, Physica D 3326 (1988)

13. M.V. Berry and S. Klein, J. Mod. Opt. 432139 (1996)

14. M.V. Berry, J. Phys. A 296617 (1996)

15. J.H. Hannay and M.V. Berry, Physica 1D 267 (1980)

16. F. Grossmann, J.M. Rost and W.P. Schleich, Z. Phys. D. 41139 (1997)

17. R. Arvieu and P. Rozmej, J. Phys. A 32 (1999) in print, (quant-ph/9810024)

18. E.N.Shurshikov, Nucl. Data Sheets 53601 (1988) 24.

19. P.M. Felker and A.H. Zewail, J. Chem. Phys. 862460 (1985)

20. J.S. Baskin, P.M. Felker and A.H. Zewail, J. Chem. Phys. 862483 (1985)

21. P.M. Felker, J.S. Baskin and A.H. Zewail, J. Phys. Chem. 90724 (1986)

22. B.C. Stipe,M.A. Rezaei and W. Ho, Science 2791907 (1998)

23. J.K. Gimzewski, C. Joachim, R.R. Schlitter, V. Langlais, H. Tang and I. Johannsen, Science 281531 (1998)

24. W.S. Warren, H. Rabitz and M. Dahleh, Science 2591581 (1993)

25. L. Shen and H. Rabitz, J. Phys. Chem. 951047 (1991)

26. J. Ortigoso, Phys. Rev. A 574592 (1998)

27. M. Persico and P.Van Leuven, Z. Phys. D 41139 (1997)

28. S. Brouard and J.G. Muga, Phys. Rev. Lett. 812621 (1998)

High resolution figures (*.tar.gzip) can be obtained as MIME attachments by e-mail from rozmej@tytan.umcs.lublin.pl 
Fig. 1. Time evolution of circular, asymmetric (left) and symmetric (right) WP with $N=14$ in the 'carpet' representation. The $|\Psi(\theta, \phi, t)|^{2}$ is presented in the contour plot for fixed $\theta=\pi / 2$.

Fig. 2. Shapes of circular, asymmetric WP in the initial stages of the evolution and some fractional revival times. Note different vertical scales used in order to show details.

Fig. 3. The same as in Fig. 2 but for symmetric WP.

Fig. 4. 'Carpet' representation of the time evolution of linear, asymmetric (left) and symmetric (right) WP with $N=110$ and $\eta=0$. In this case the probability density $2 \pi \sin \theta|\Psi(\theta, t)|^{2}$ is presented in the contour plot.

Fig. 5. Shapes of clones and mutants for asymmetric (left) and symmetric (right) WP with $N=14$ and different $\eta$ at time $t=T_{\text {rev }} / 8$. The $|\Psi(\theta, \phi, t)|^{2}$ is presented in spherical coordinates as the radial coordinate, $\theta$ is an angle with respect to $O x$ axis which is the axis of cylindrical symmetry in this case. (Only upper part of the probability density is displayed)

Fig. 6. Time evolution of nulear rotational wave packet obtained in $\mathrm{CE}$ of ${ }^{238} \mathrm{U}$ presented in 'quantum carpet' representation. Contours of $2 \pi \sin \theta|\Psi|^{2}$ are plotted.

Fig. 7. Shapes of nulear rotational wave packet obtained in $\mathrm{CE}$ of ${ }^{238} \mathrm{U}$ during a short time evolution.

Fig. 8. The same as in Fig. 7 for some particular much longer times. 
$\mathrm{N}=14 \quad \eta=1$ all I

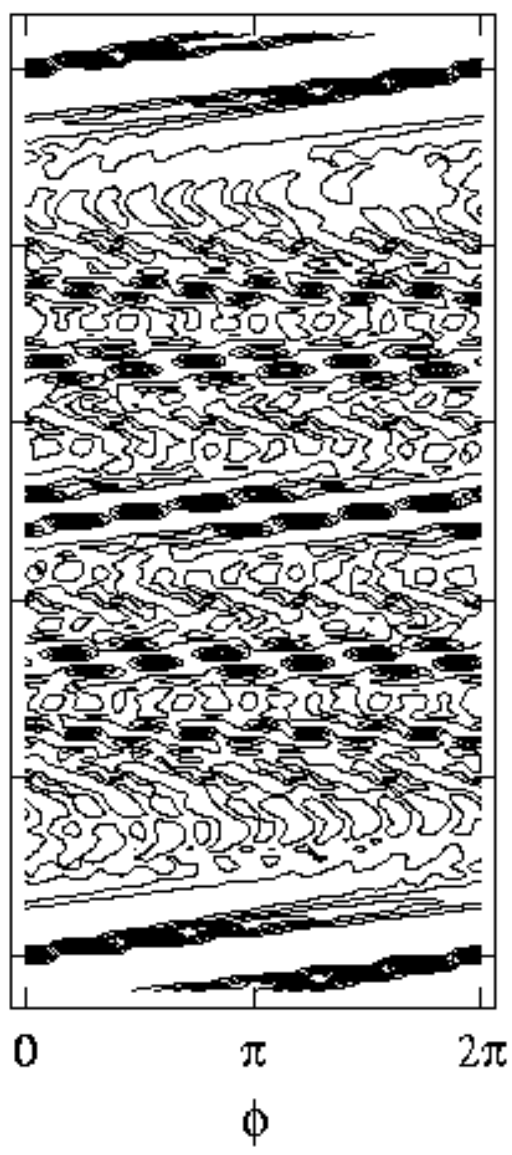

$\mathrm{N}=14 \eta=1$ even I

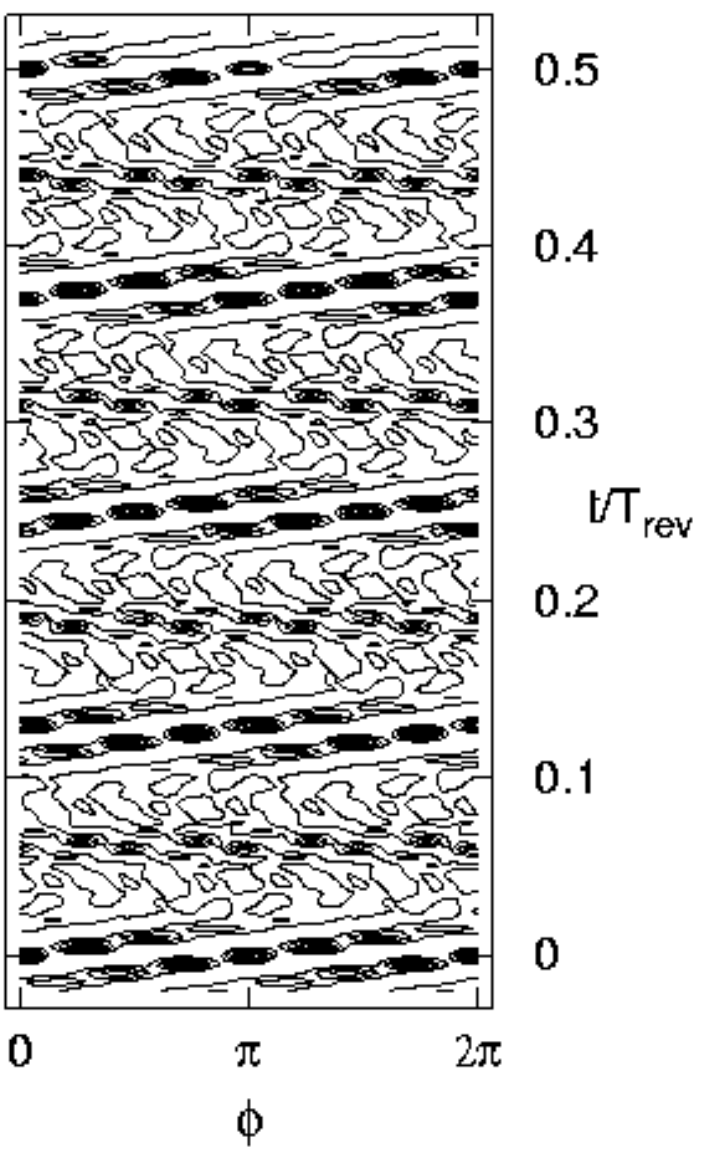

Fig. 1. Time evolution of circular, asymmetric (left) and symmetric (right) WP with $N=14$ in the 'carpet' representation. The $|\Psi(\theta, \phi, t)|^{2}$ is presented in the contour plot for fixed $\theta=\pi / 2$. 

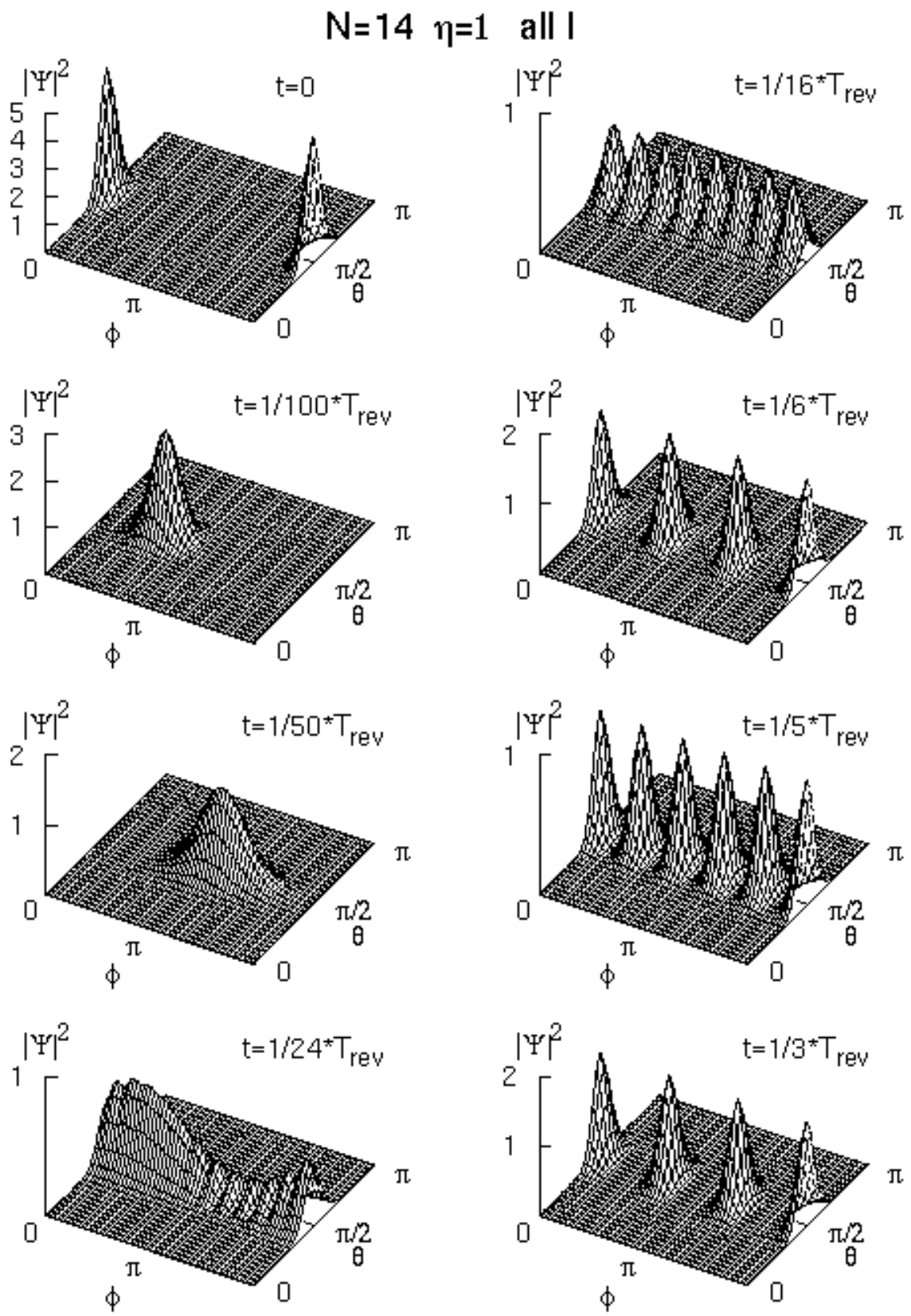

Fig. 2. Shapes of circular, asyrnmetric WP in the initial stages of the evolution and some fiactional revival times. Note different vertical scales used in order to show details. 

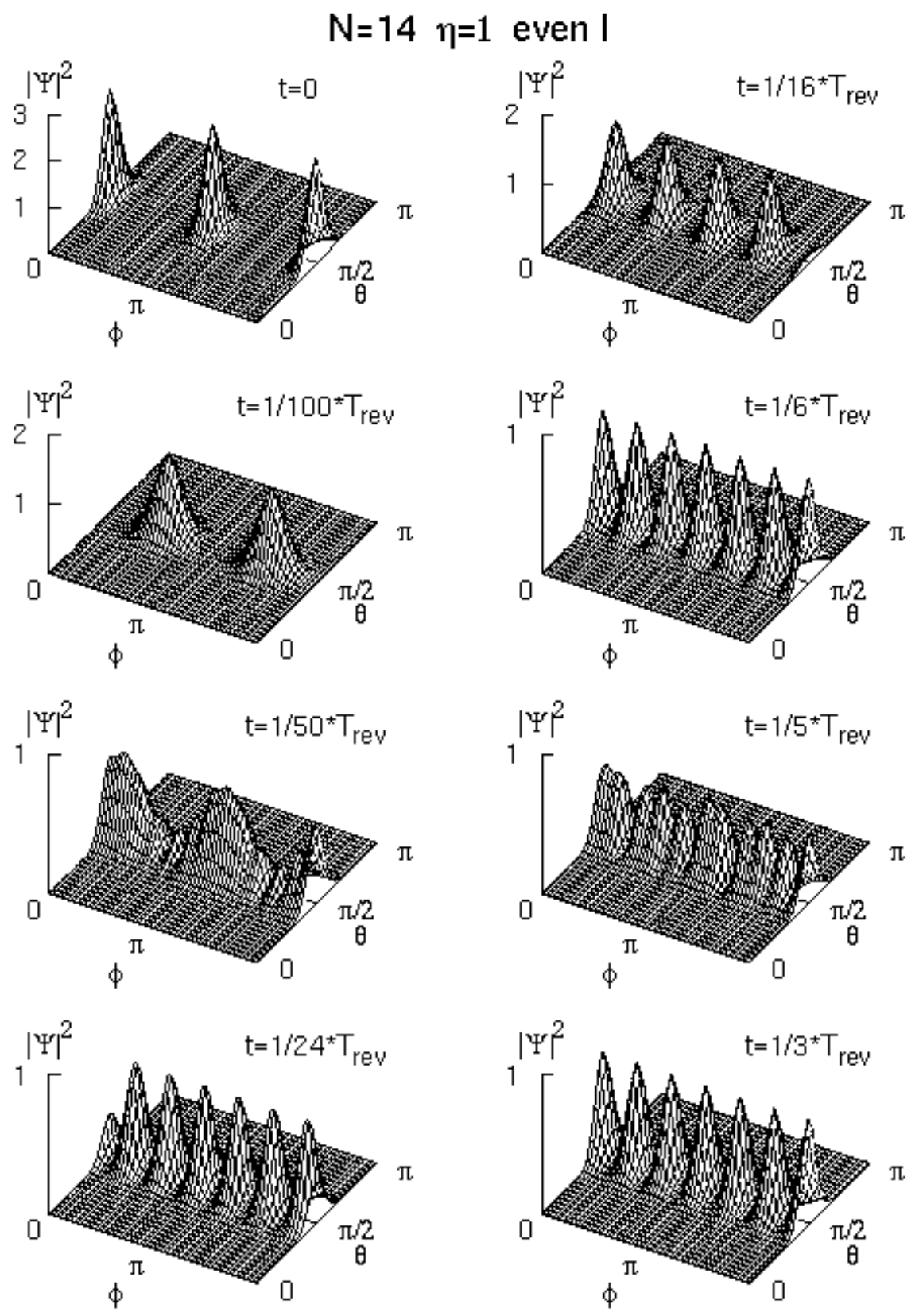

Fig. 3. The same as in Fig. 2 but for symmetric. WP. 


\section{$N=110 \quad \eta=0$ all I $\quad N=110 \quad \eta=0$ even I}
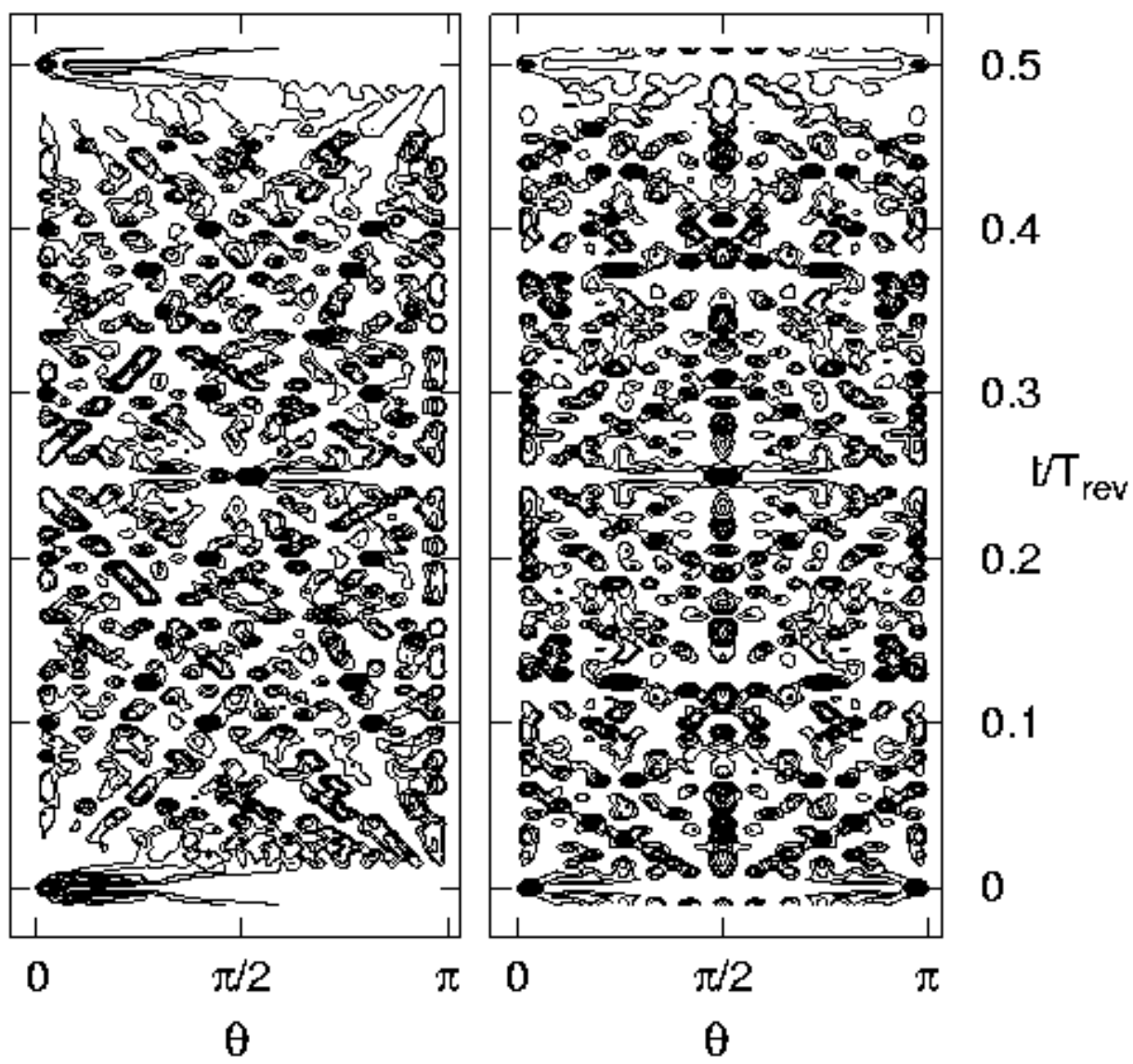

Fig. 4. 'Carpet' representation of the time evolution of linear, asymmetric (left) and symmetric (right) WP with $N=110$ and $\eta=0$. In this case the probability density $2 \pi \sin \theta|\Psi(\theta, t)|^{2}$ is presented in the contour plot. 

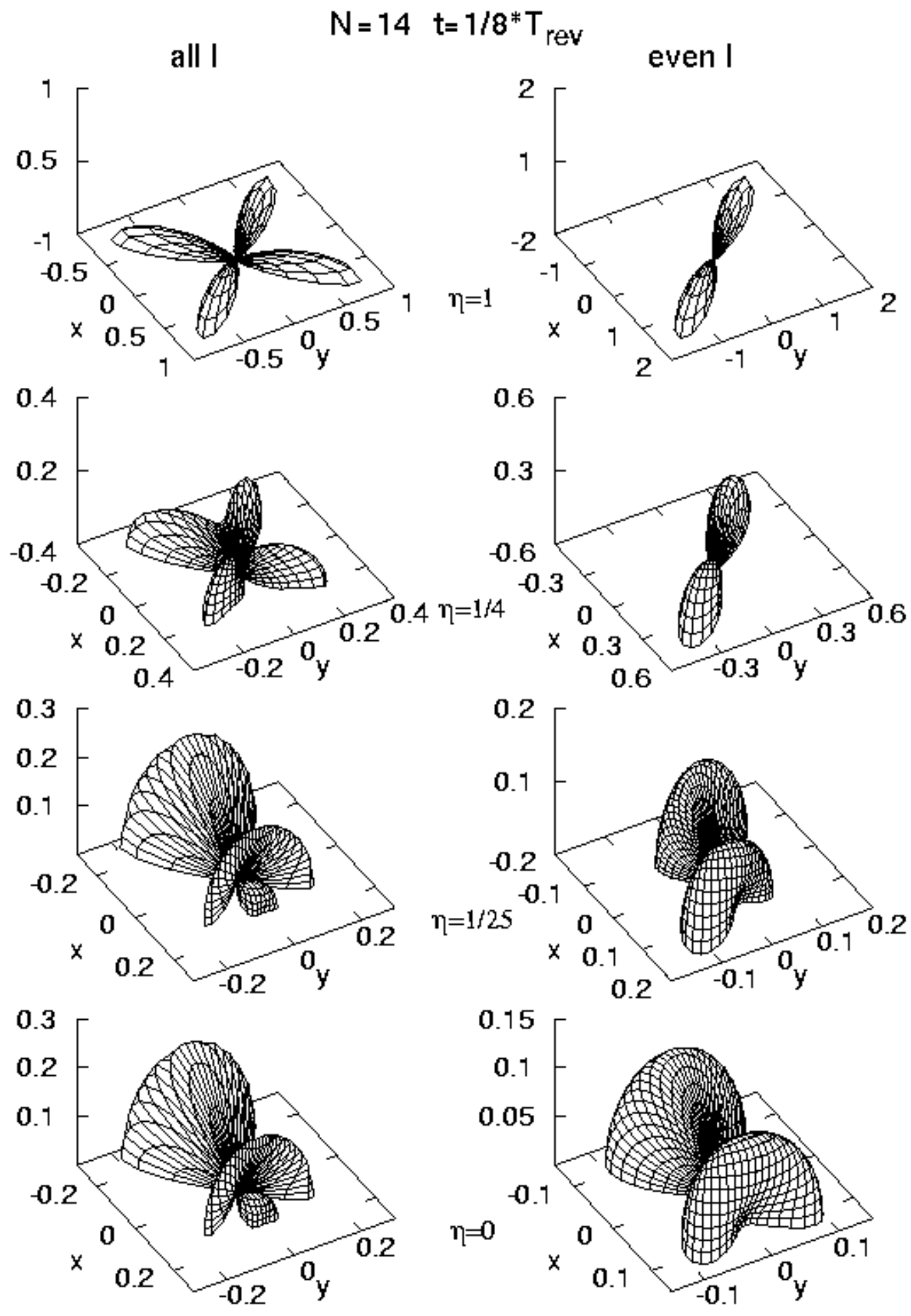

Fig. 5. Shapes of clones and mutants for asymmetric (left) and symmetric (right) WP with $N=14$ and different $\eta$ at time $t=T_{\text {rev }} / 8$. The $\mid\left.\Psi(\theta, \phi$ : $t)\right|^{2}$ is presented in spherical coordinates as the radial coordinate, $\theta$ is an angle with respect to $D x$ axis which is the axis of cylindrical symmetry in this case. (Only upper part of the probability density is displayed) 


$$
E_{1}=\mid(\mid+1) \quad \text { Exp. energies }
$$

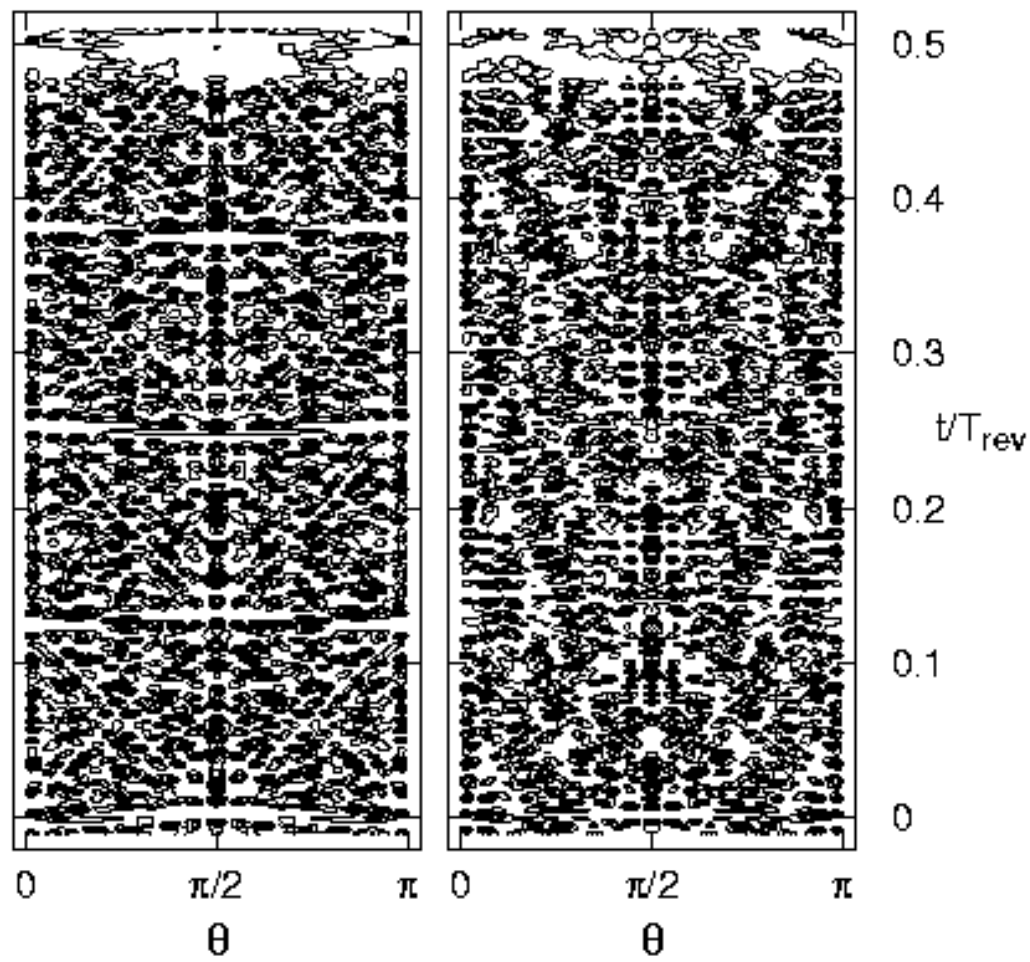

Fig. 6. Time evolution of nulear rotational wave packet obtained in $\mathrm{CE}$ of ${ }^{228} \mathrm{U}$ presented in 'quanturn carpet' representation. Contouls of $\left.2 \pi \sin \theta||^{2}\right|^{2}$ are plotted. 


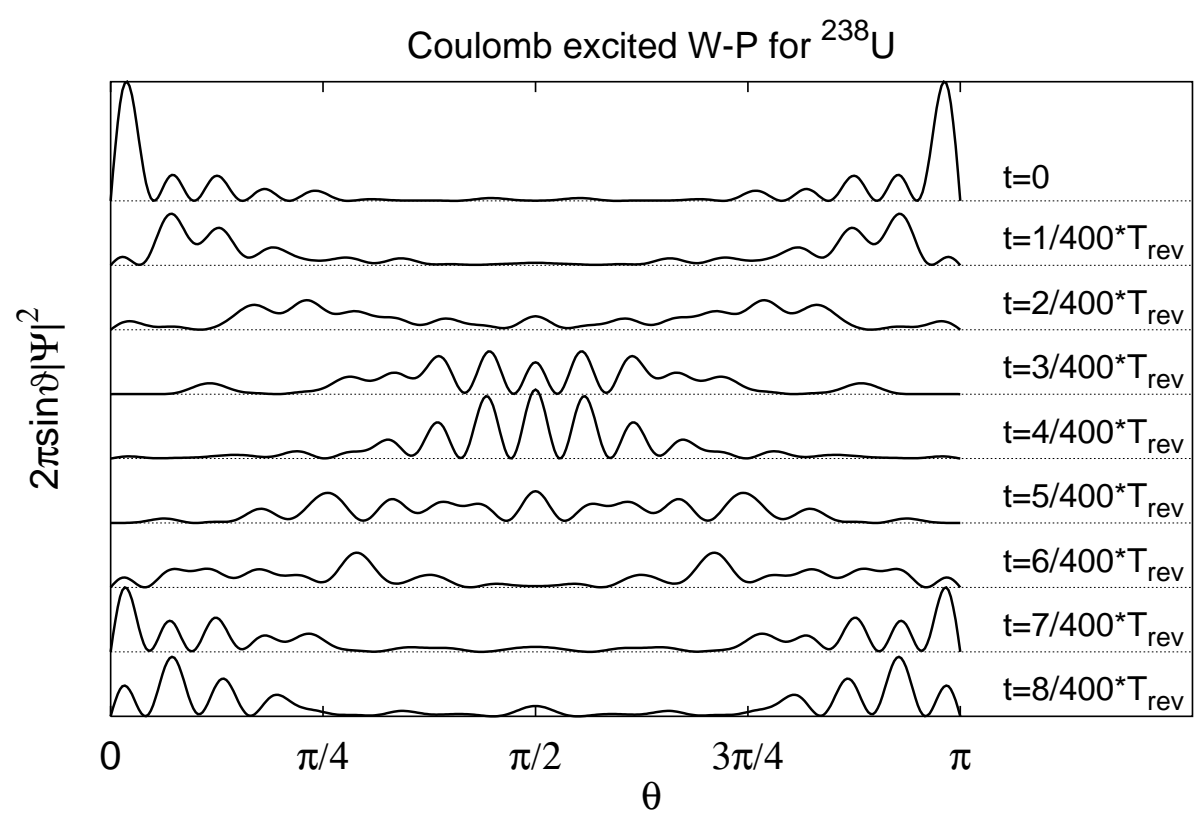

Fig. 7. Shapes of nulear rotational wave packet obtained in $\mathrm{CE}$ of ${ }^{238} \mathrm{U}$ during a short time evolution. 


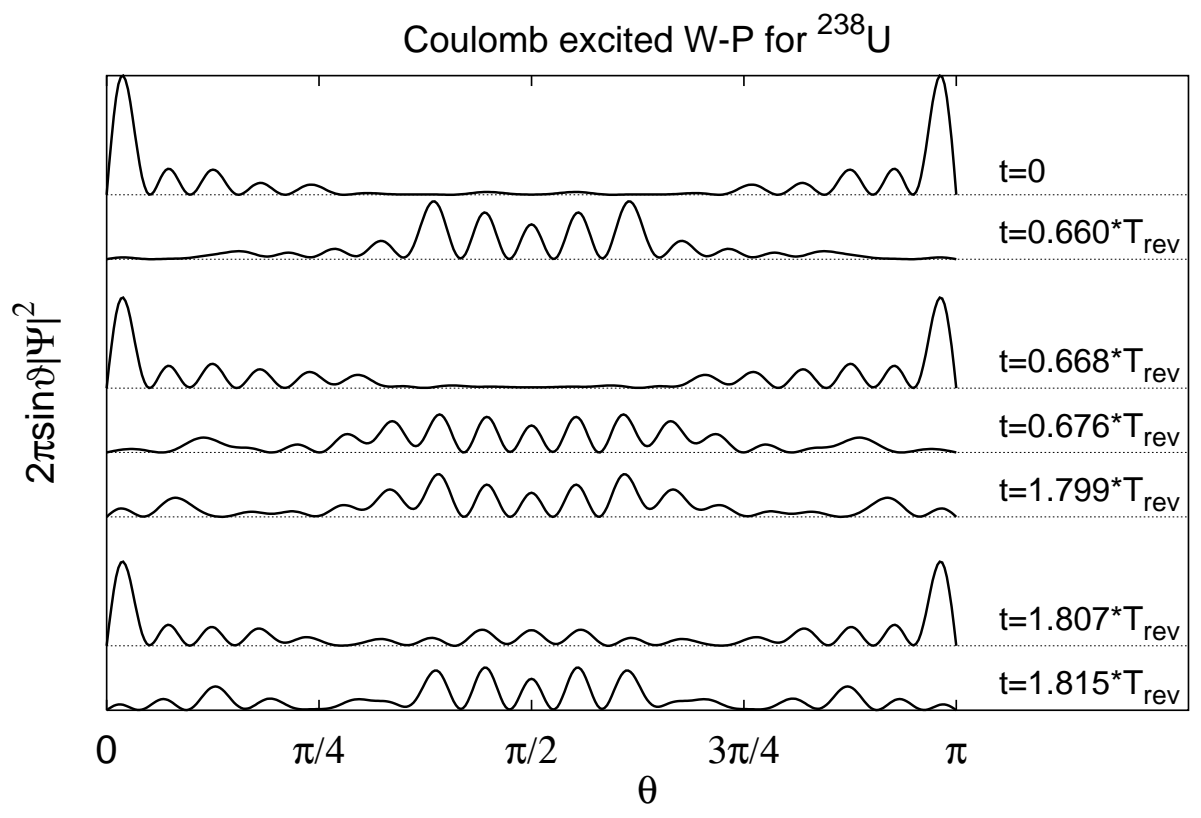

Fig. 8. The same as in Fig. 7 for some particular much longer times. 